\title{
Uma ontologia para auxílio em ambientes colaborativos para busca de informações
}

\author{
João P. R. P. da Silva ${ }^{1,2}$, José Maria Nazar David ${ }^{2}$, \\ Eduardo Barrere $^{1,2}$, Jairo Francisco de Souza ${ }^{1,2}$ \\ ${ }^{1}$ LApIC Research Group - Instituto de Ciências Exatas \\ Universidade Federal de Juiz de Fora \\ 36.036-900 - Juiz de Fora - MG - Brasil \\ ${ }^{2}$ Programa de Pós Graduação em Ciência da Computação \\ Universidade Federal de Juiz de Fora \\ 36.036-900 - Juiz de Fora - MG - Brasil \\ \{jpradd, jose.nazar, eduardo.barrere, jairo.souza\}@ice.ufjf.br
}

\begin{abstract}
Search engines on the web are a source of study and learning. Searching as Learning (SaL) and Collaborative Information Seeking (CIS) are areas that analyze learning as a process, the second being collaborative. The literature demonstrates the need for more studies in these areas and better mapping of student learning. This work presents CollabSO, an ontology that models the experimental context in collaborative search environments, something not yet explored for CIS. In addition, it support for persistence, coordination and cooperation of student groups. The ontology aims to support pedagogical analysis, teacher action and execution of CIS experiments.
\end{abstract}

Resumo. Os mecanismos de busca na web são fonte de estudo e aprendizado. Searching as Learning (SaL) e Collaborative Information Seeking (CIS) são áreas que analisam o aprendizado como um processo, sendo o segundo de forma colaborativa. A literatura demonstra a necessidade de mais estudos nessas áreas e melhor mapeamento do aprendizado dos alunos. O presente trabalho apresenta a CollabSO, uma ontologia que modela o contexto experimental em ambientes de busca colaborativa, algo ainda não explorado para CIS. Além disso, fornece suporte para persistência, realização da coordenação e cooperação dos grupos de alunos. A ontologia visa dar suporte para análise pedagógica, ação dos professores e execução de experimentos CIS.

Palavras-chave: Ontologia, Collaborative Information Seeking, Collaborative Search, Searching as Learning

\section{Introdução}

Com os avanços da internet, várias áreas ganham força, como a Web Semântica e a Educação online. Com isso, diversas técnicas, modelos e pesquisas são necessárias para aperfeiçoar a eficiência e obter melhores resultados na aprendizagem e engajamento dos alunos. A internet é hoje uma das fontes mais utilizadas para se buscar por informações 
e as ferramentas de busca têm investido em abordagens para gerar resultados mais eficientes. Tais ferramentas podem funcionar bem para certos tipos de tarefas básicas de pesquisa, mas não suportam tarefas mais complexas como, por exemplo, busca para aprendizado e busca para investigação. Em outras palavras, as ferramentas de busca estão mais otimizadas para auxiliar em aprendizado factual do que em outros tipos de aprendizados, como entendimento, análise, aplicação ou síntese [Fulantelli et al. 2016].

Desde a intenção de se buscar uma informação como aprendizado até o momento que se obtém o conhecimento desejado, acontece um processo de aprendizagem com a busca [Machado et al. 2019]. Searching as Learning (SaL) é a área que visa investigar a profunda relação entre o processo de busca e a aprendizagem [Vakkari 2016]. No processo de $\mathrm{SaL}$, os estudantes possuem rendimentos bem diferentes, principalmente quando existe o fator de conhecimento prévio do assunto [Karanam and van Oostendorp 2016, Chi et al. 2016]. Com isso, existem alunos realizando um esforço em um processo de busca pouco eficiente até obterem algum domínio do assunto [Mao et al. 2016]. Sendo assim, torna-se necessário: desenvolver um sistema para apoiar o processo de aprendizagem; apoiar uma interação eficaz do usuário para pesquisar como aprendizado; fornecer ferramentas de alfabetização baseada em inquérito dentro de um sistema de pesquisa; e avaliar a aprendizagem do comportamento de pesquisa on-line [Rieh et al. 2016]. Os usuários devem ser acompanhados e orientados sobre o processo de pesquisa à medida que o processo de aprendizagem ocorre [Machado et al. 2020]. É comum existir um desconhecimento prévio e incerteza da informação, chamado de Anomalous State of Knowledge (ASK), relacionado à incapacidade de especificar com precisão suas necessidades de informação [Tibau et al. 2019].

Collaborative Information Seeking (CIS) é uma área com algumas convergências a SaL, porém trabalha nesse contexto com busca em grupo. Em [Rikarno Putra 2018], por exemplo, ficou demonstrado um maior ganho de aprendizagem por colaboração em atividades de busca de informação em comparação com a individual. Recursos colaborativos podem ajudar a comunicação no processo de busca, mas a existência deles não garante que a colaboração ocorra com qualidade [Mayweg-Paus et al. 2020]. Dado esse problema, o presente trabalho objetiva em apresentar um apoio à experimentos CIS com a Collaborative Searching Ontology (CollabSO), uma ontologia para a busca colaborativa de informações na web. CollabSO incorpora informações sobre troca de mensagens, documentos compartilhados, acesso a histórico de busca de usuários, fóruns, coordenação e organização de grupos. A ontologia contém recursos de proveniência de dados da PROVO [Lebo et al. 2013], pois a proveniência ajuda na documentação de todo o processo de busca e aprendizado para a análise dos professores. Sobre a intervenção e a relação do professor no aprendizado dos alunos é apresentado por [van Leeuwen and Janssen 2019] nos seguintes pontos: foco do aluno reflete o do professor; estimular discussão gera bons resultados; colocar alunos para explicar aos demais gera ganho de conhecimento; equilíbrio na presença do professor; tecnologias de análise são necessárias para rastrear interação complexa de colaboração; professor que acompanha não é um professor passivo, mas que age quando necessário. No presente artigo, com a ontologia, será possível rastrear, apoiar e apresentar aos professores informações para interagir com os alunos solucionando os pontos citados.

Como constatado por autores sobre SaL [Hoppe et al. 2018, Vakkari 2016, 
Rieh et al. 2016, Machado et al. 2020] e CIS [Hertzum and Hansen 2019, Hong et al. 2019, Avià 2019], existe um grande volume de experimentos laboratoriais, mas essas áreas carecem de modelos para avaliar a aprendizagem e identificar comportamentos. A revisão de [Mayweg-Paus et al. 2020] demonstra que na maioria dos sistemas CIS a coordenação ocorre sem ser guiada, flexível ou sem ser realizada. Destaca-se a necessidade de estudos sobre a coordenação e sobre a influência dos agentes pedagógicos no processo de aprendizagem. Com a ontologia proposta, busca-se facilitar a análise, estudo e realização de experimentos. A contribuição desse modelo supre as necessidades documentadas na literatura, relacionada à aprendizagem por busca no âmbito educacional de SaL e CIS.

Esse trabalho está estruturado da seguinte forma: a seção 2 apresenta a Fundamentação Teórica, e a seção 3 discute os trabalhos relacionados com a colaboração, ontologias e pesquisa web. Em seguida, na seção 4 é apresentada a proposta da ontologia detalhando a sua estrutura e como esta pode ser utilizada. Na seção 5 apresentam-se as conclusões e trabalhos futuros.

\section{Fundamentação Teórica}

A área de $\mathrm{SaL}$ estuda a sinergia entre os processos de busca e aprendizagem [Vakkari 2016], tratando tais processos como um processo humano [Machado et al. 2019]. Muito tempo de pesquisa nas tarefas de aprendizagem é dedicado à análise e comparação de resultados, bem como à reformulação de consultas [White and Roth 2009]. Para se construir uma ferramenta sobre SaL é necessário que a mesma possa auxiliar os usuários em suas tarefas de aprendizado com processos adaptados de ranqueamento e recuperação de informação [Hoppe et al. 2018].

CIS é um processo de pesquisa social no qual os usuários possuem necessidades de informação em comum e trabalham ativamente em conjunto para cumpri-lo [Morris and Teevan 2009]. Os usuários colaboram sobre um assunto que não possuem conhecimento individual suficiente, e obtêm ajuda mútua para um aprendizado pessoal [Wu et al. 2018]. CIS oferece oportunidade aos alunos para uma variedade de recursos e perspectivas através de diálogos e análises dos materiais que têm forte relação com o sucesso na aprendizagem [Knight et al. 2017].

A aprendizagem colaborativa é multidisciplinar e envolve áreas como Educação, Psicologia, Sociologia, Ciência da Computação, entre outras [Wu et al. 2018]. O trabalho de [Reynolds 2016] destaca a relação entre processo e aprendizagem, envolvendo a qualidade das strings de consultas, a colaboração na busca e no desenvolvimento das atividades. Há espaço para análise mais aprofundadas da influência da área de CIS nos efeitos da aprendizagem, algo que não tem sido muito explorado em SaL [Wu et al. 2018].

Colaboração no aprendizado é algo que exige planejamento, coordenação, implantação de currículo pedagógico e tecnologias apropriadas [Stahl et al. 2006]. Com isso, ontologias podem auxiliar a geração de um modelo para aprendizagem social no ensino para apoiar a colaboração [Oktavia et al. 2016]. A organização do conhecimento e a modelagem de dados podem ser obtidos pela representação de uma ontologia [Basu 2019] e a geração desses modelos facilitam a reutilização através de padrões e evitam um trabalho maior na criação do zero [Souza et al. 2016]. As ontologias podem ser utilizadas no apoio de experimentos e análises de comportamento, pois as ontolo- 
gias são capazes de modelar as relações dos alunos e dos recursos de aprendizagem, sendo úteis em recuperar detalhes [Konys 2018]. O uso da PROV-O nesse processo se torna algo interessante por fornecer um conjunto de classes, restrições e propriedades podendo ser via de representação e interpretação de proveniência entre contextos e sistemas [Lebo et al. 2013].

\section{Trabalhos Relacionados}

No universo de aprendizagem pela busca, poucos são os trabalhos com o uso de ontologias ou que modelam um experimento CIS. Por exemplo, [Tibau et al. 2018] faz uso da ontologia Knowledge Intensive Process Ontology (KIPO) para explorar elementos do conhecimento tácito do processo de aprendizagem. O trabalho apresenta um modelo de busca exploratória na web na formação de professores de línguas, evoluindo e validando o modelo. Com isso, foi possível a visualização do padrão de busca e aprendizagem, porém, os autores não exploraram a busca colaborativa. [Oktavia et al. 2016] identificaram fatores de aprendizagem social que permitem ao ensino superior ser sustentável e adaptável às mídias sociais. O resultado desta pesquisa é uma ontologia baseada no Zachman Framework [Zachman 1987] que pode ser implementada para educação de ensino superior, porém também não foca em experimentos CIS.

Em [Alabri et al. 2019], é discutida uma mineração de dados das conversas de aluno em chats. As mensagens são analisadas através de uma ontologia para extrair as relações semânticas a fim de construir o modelo de domínio e gerar mais informações para personalização de conteúdo em ambiente de e-Learning. Dessas conversas, desejou-se descobrir estilos de aprendizagem e o nível de conhecimento dos alunos. Com avanços em estudos sobre o processamento de linguagem natural (PLN) ou sobre a mineração de argumentos, surgem novas possibilidades de identificar e classificar comportamento, como ocorre em [Dionísio et al. 2017]. Esse estudo realiza uma abordagem de classificação automática em fóruns educacionais (presença de grupo, elogios, compartilhamento de informações e novas perspectivas de postagens colaborativas). A abordagem utilizada serve para auxiliar professores no acompanhamento dos alunos. Esse tipo de trabalho pode enriquecer análises de comportamento em experimentos CIS associados a ontologias.

Por sua vez, a ontologia CONTO [Souza et al. 2016] serviu de base para a geração de Padrões de Ontologia (PO) de colaboração que seguem o modelo 3C: Cooperação, Comunicação e Coordenação. Para executar essa tarefa, os autores utilizaram o OntoUML lightweight editor (OLED) com extensão para uso do Domain-related Ontology Pattern (DROP). Já o MEMORAe- Collaboration-Context (MCC) [Li et al. 2019] contribui para esclarecer as características de uma colaboração e seu contexto em diferentes fases: preparação, a colaboração e debriefing. O MCC foca em representar o contexto na ontologia, seja dos eventos que ocorrem em um dado período de tempo, quanto das informações dos envolvidos nas atividades de cooperação. O CONTO é generalista sobre colaboração, mas pouco específica para experimentos CIS, foco do presente artigo.

Existem também ontologias que auxiliam a estruturação, entendimento e funcionamento de sistemas de cursos remotos. Algumas ontologias fornecem informações dos alunos ajudando a intervenção dos docentes, em alguns casos, são apoiadas na Taxonomia de Bloom. [Hu et al. 2019] apresenta um modelo de Tecnologia da Informação em 
e-Learning Systems baseado em ontologia. O modelo permite auxiliar os professores em suas atividades, ganhando tempo e promovendo flexibilidade para melhorar conteúdos e atividades nos ambientes de aprendizado.

Existem ontologias relacionadas ao aprendizado com busca ou com atividades colaborativas. No entanto, neste trabalho é apresentada uma ontologia específica para CIS, o que não foi explorado na literatura. Nos últimos anos foram apresentados trabalhos com soluções importantes para colaboração, mas que ainda não se aplicaram no contexto de busca colaborativa, ou existe uma necessidade de investigações para CIS. No presente trabalho, exploram-se os seguintes pontos: a identificação de contexto como em [Li et al. 2019] e de fácil reutilização como o CONTO, mas para a busca colaborativa com o PROV-O [Lebo et al. 2013]; a criação de estruturas de comunicação que permitiriam análises de mineração de argumento como em [Alabri et al. 2019] e [Dionísio et al. 2017] caso associadas à CollabSO; a identificação e especificação sobre a coordenação, como destacado ser necessário em [Mayweg-Paus et al. 2020] e que é negligenciado pelos pesquisadores; a criação de estrutura que mapeia e gera possibilidades de análise de $e$ Learning pelos professores como os outros trabalhos listados destacaram como fator importante no aprendizado. ColabSO é uma ontologia objetiva e abrangente naquilo que tem sido destacado na literatura como importante no contexto de colaboração. Objetiva em especial para CIS, no qual se torna inovadora por não possuir ontologias que explorem esse processo de aprendizado.

\section{Ontologia Proposta: CollabSO}

Individualmente, os usuários geralmente possuem conhecimentos ou habilidades insuficientes para resolver a tarefa e, por um CIS, podem não só aprender individualmente como podem se ajudar por uma colaboração [Chi et al. 2016]. Com isso, esse trabalho propõe uma ontologia que modela a colaboração através de uma tarefa de busca de informações na web. Esse modelo é importante para entender os relacionamentos que ocorrem e melhorar a compreensão de como o aprendizado ocorre. O modelo possibilita estruturar experimentos no contexto de CIS e SaL e auxilia professores em atividades práticas através de análises semânticas de coleta de informações do estudo dos alunos e gestão dos mesmos.

Ao analisar redes colaborativas, existe o desejo de saber quem aprende, o quê, com quem, onde, quando e em que circunstâncias [Haythornthwaite 2019]. O modelo ontológico pode facilitar a observação de como esses eventos ocorrem em sua estrutura. Lembrando que é importante o professor observar e motivar as relações dos alunos e a se responderem [Hiltz and Turoff 2002]. No modelo são definidas relações aluno-professor, possibilitando registro e análise pedagógica. Os objetos de aprendizagem estão representados sobre o contexto de busca e compartilhamento de informações tal como ocorre na literatura de CIS. Também estão organizados elementos sobre passos de desenvolvimento e coordenação dos grupos de alunos, como será apresentado mais a frente.

A encenação das competências ocorre através da representação de classes de aluno e professor que são agentes dentro do PROV-O. A ontologia representa o contexto de aprendizado colaborativo facilitando as verificações necessárias para entender a dinâmica da aprendizagem, representando os grupos, a coordenação e o mapeamento da execução das atividades. Conhecer, avaliar, modelar e aperfeiçoar ambientes de aprendizado, em 
especial de Searching as Learning e Collaborative Learning, são campos de grande necessidade e possibilidades de atuação. Na subseção seguinte, a estrutura e os componentes da ontologia serão apresentados e detalhados.

\subsection{Estrutura da Ontologia}

A estrutura da ontologia foi baseada na PROV-O, destacando suas três principais classes: Entity, Agent, Activity. A primeira é "uma coisa física, digital, conceitual ou outro tipo de coisa com alguns aspectos fixos; entidades podem ser reais ou imaginárias" [Lebo et al. 2013]. Uma subclasse da Entity é a Collection, sendo uma estrutura que constitui as entidades. No contexto CIS, foram inseridos na ontologia os Recursos Colaborativos (chat, fórum, histórico de busca e documentos compartilhados). Os recursos possuem componentes (log de histórico, $\log$ de chat, termos de busca e tópico de fórum) também inseridos como subclasse de Collection. Plan é outra entidade com sua subclasse Step.

O Agent possui a subclasse Person que engloba as classes de professor, aluno e Organization, a classe que representa os grupos. Agent trata de todo ator com responsabilidade de realização de uma atividade através de uma entidade. A Activity é algo que contém um limite temporal que atua sobre as entidades. Logo, a classe Task foi atribuída como subclasse da Activity, significando a tarefa de objetivo do estudo/experimento CIS. Coordination também é uma atividade a ser executada pelos alunos coordenadores dos grupos. A Figura 1 mostra a estrutura de algumas classes e seus relacionamentos mais básicos.

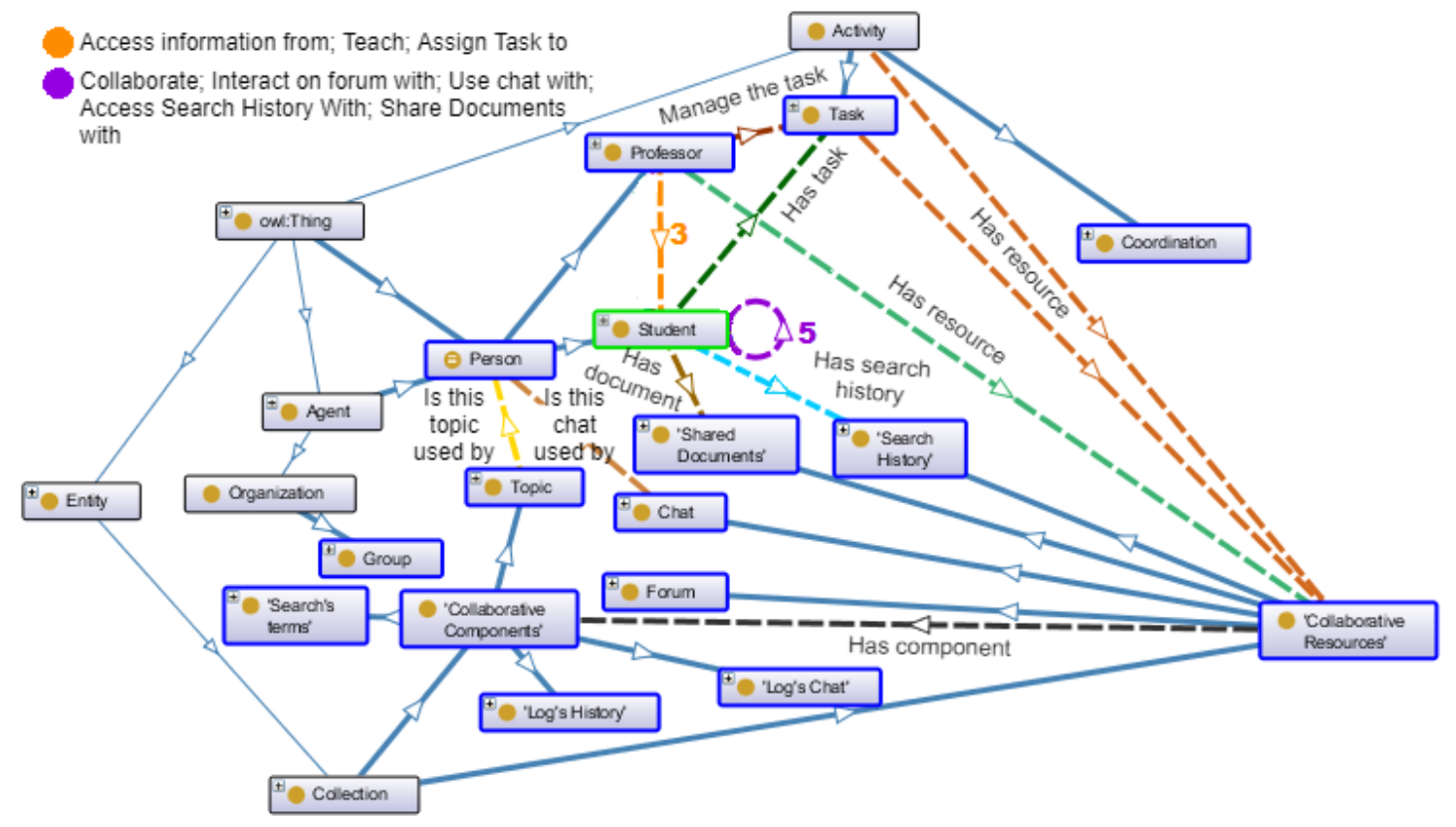

Figura 1. Principais classes da ontologia

A Figura 1 mostra setas azuis contínuas referente às subclasses, e as coloridas pontilhadas são referentes às object properties. Esta segunda mostra as regras de relacionamento entre todos os indivíduos e a imagem mostra apenas algumas delas para facilitar a visualização. Além de todas as relações já contidas no PROV-O, que são especificadas 
na ontologia, temos relações de acesso de informações como histórico de busca de outros alunos, interações de fórum, uso de chat, compartilhamento de documentos, atribuição de tarefa, ensinar, criação de revisões (feedback de tarefas, relatórios e avaliação), moderação e uso de recursos, relações de composição e pertencimento. Relações de coordenação, influência, tempo e qualificação, por exemplo, são feitas pelo próprio PROV-O. A ontologia também conta com super properties (chain), gerando relacionamentos de propriedade por questões condicionais (para dois alunos compartilharem um documento, é necessário que um deles o possua, por exemplo). A Figura 1 mostra diversas classes originais do PROV-O, que foram reutilizadas integralmente e permitem o processo de mapeamento de proveniência. Para diferenciar as classes adicionadas com as classes da estrutura da PROV-O, elas foram ilustradas com contorno azul em destaque.

Na Figura 1 estão representadas apenas algumas das classes originais do PROV-O e a maioria das classes criadas com parte das object properties. A ontologia está disponível ${ }^{1}$ para melhor visualização de toda a estrutura em mais de uma opção, o grafo e a própria ontologia em sintaxe $O W L$.

\subsection{Exemplos de aplicação}

O uso de experimentos para analisar ambientes SaL e CIS são frequentes e importantes para pesquisa em educação. A CollabSO proporciona um contexto que facilita experimentos de busca colaborativa com formação de grupos de qualquer tamanho fornecendo os dados de histórico de busca, documentos e interação de chat entre esses alunos. A ontologia também comporta experimentos que façam uso de fóruns e criação de tópicos. Esse modelo representa o professor e sobre acesso de informações, interação e controle das atividades dos alunos, acompanhando suas atividades em seus grupos, relacionando com alunos dentro e fora de seu círculo. A ontologia não está limitada a possibilidade de uso somente em experimentos, mas pode ser usada como suporte de desenvolvimento de um sistema e-learning colaborativo com foco e processos de aprendizagem com busca. Como possui um suporte de proveniência fornecido pela PROV-O que segue os padrões da W3C, pode contribuir nas definições de contexto, diversas análise e manipulações de dados.

Para descrever uma situação real CIS associada à ontologia, segue o caso de uma turma de alunos da professora de nome Regina, a qual leciona (Teach) para o John e lhe atribui tarefas (Assign task to), tendo acesso às suas informações (Access information from). John, por sua vez, tem acesso ao documento "DOC1" (Has document), ao histórico de busca "SH1" (has search history) e interage no fórum no tópico "TOPIC1" (Is this topic used by). John é coordenador (C1) do grupo "Group1" com Gaspar, com o qual compartilha o documento (Share documents with), histórico (Access search history with) e se comunicam no chat (Use chat with). A sua coordenação está associada à atividade "Atividade1", que foi associada a John (wasAssocietedWith) e ele deve compri-la (Has Task). O grupo de John executou as etapas "ST1" e "ST2" de desenvolvimento (hadPlan) que são iniciadas e terminadas pela coordenação "Coord1"(wasInfluencedBy). Frank é aluno de outro grupo que também é coordenador e interage com John pelo fórum (Interact on forum with). Com o compartilhamento de recursos e chats, os alunos realizam a colaboração trocando conhecimento da busca e realizando os objetivos da atividade.

\footnotetext{
${ }^{1}$ https://drive.google.com/drive/folders/1tfcaGj6Y_ A5oPoSbECWQmAo-bkpp 4xwW?usp=sharing
} 


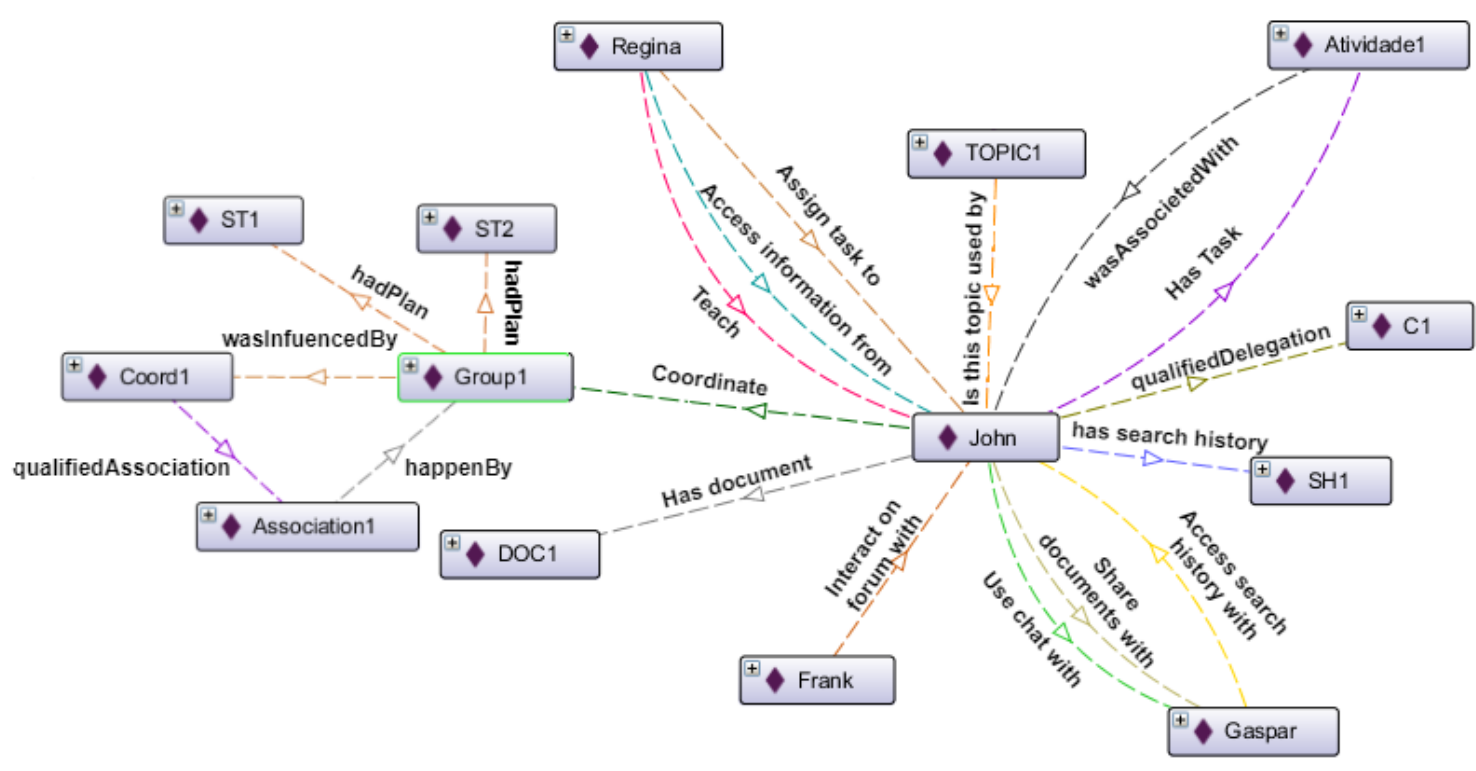

Figura 2. Situação de atividade na CollabSO com foco em "John"

Na Figura 2, é representada a situação descrita na ontologia. A representação está focada na situação de John, mas se o todas as ligações estivessem representadas, teríamos mais ligações como com Regina e outros alunos, além dos dados de Frank com a "Atividade1", por exemplo.

A CollabSO é uma ontologia que mapeia todo o processo de aprendizagem e representa a colaboração em um aprendizado com busca. A ontologia é consistente em representar experimentos CIS, proporcionando ao professor uma estrutura de proveniência e dados para análise e conhecimento. CollabSO resolve a falta de uma ontologia de CIS, está configurada com as características destacadas pela literatura como importante na investigação e estudo nessa área de busca de informações online

\section{Conclusão}

Nesse trabalho, foram apresentados diversos estudos sobre SaL e CIS mostrando a necessidade de se acompanhar experimentos de aprendizagem com busca em grupos. Através de uma revisão, foram levantados pontos importantes do estado da arte sobre busca colaborativa e de trabalhos relacionados sobre ontologias. Com isso, o presente trabalho apresenta uma ontologia no domínio de CIS com pontos positivos usados na literatura relacionados à busca no aprendizado e pontos pouco explorados até mesmo nos experimentos CIS, mas que já eram explorados em outros contextos de aprendizagem colaborativa. Sendo assim, a ontologia se torna um diferencial ao representar conceitos pertinentes, especificamente, ao domínio de CIS, algo que não foi ainda explorado em ontologias de aprendizagem com busca colaborativa. A ontologia CollabSO incorpora mapeamento de proveniência com o PROV-O, facilitando a documentação e análise dos acontecimentos do aprendizado na busca. A CollabSO incorporou os elementos de CIS na sua estrutura com conceitos de professores, alunos, grupos, histórico de busca, documentos compartilhados, chat, fórum, feedback de alunos, notas dos professores e etapas de execução da atividade. Lembrando da importância do 3C da colaboração (Coordenação, Comunicação e Cooperação), a ontologia incorporou definições de atribuição de co- 
ordenador e coordenação, algo importante e pouco explorado nos trabalhos de CIS [Mayweg-Paus et al. 2020]. Os 3C estão extremamente relacionados, sendo a cooperação executada com apoio dos meios de comunicação e organização da coordenação. A existência do professor executa um papel importante nesse meio podendo estimular os alunos a cooperarem, e acompanhar todo o processo.

Como apresentado, existe um grande volume de experimentos laboratoriais, mas essas áreas carecem de modelos para avaliar a aprendizagem e identificar comportamentos. A ontologia pode ser utilizada: associada a experimentos e atividades educacionais de busca colaborativa; como apoio para estruturar os dados de todo o processo de aprendizagem e mapeamento da persistência dos dados. A CollabSO abre a possibilidade de melhorar o conhecimento dos eventos e acontecimentos durante o aprendizado e até compreender melhor como o conhecimento é adquirido em cada etapa da busca, como descrito na revisão de [Mayweg-Paus et al. 2020] como algo necessário. O professor, com o apoio do rastreamento dos acontecimentos da atividade e comportamento dos aluno, pode moderar e motivar os alunos para que a colaboração ocorra de forma efetiva, como destacado por [van Leeuwen and Janssen 2019]. Uma vez que, a existência de elementos colaborativos não garantem a colaboração.

A ontologia abre várias possibilidades de experimentos, mas é necessário associála dentro de uma estratégia do responsável pelo experimento. Sendo assim, a CollabSO é capaz de fornecer uma estrutura para guiar um professor com a criação de um sistema de learning analytics ou para realizar toda a interação do experimento CIS. Sendo assim, a ontologia está limitada aos conceitos do estado da arte acerca do que é importante investigar e acompanhar em experimentos CIS. A ontologia ainda precisa ser aplicada dentro de experimentos de busca colaborativa.

Para elaborar a ontologia CollabSO, foram exploradas as necessidades existentes sobre as ontologias da literatura e sobre CIS. Sendo assim, poderá ser associada aos sistemas existentes e criação de novos para experimentos. Um diferencial para novas pesquisas seria a verificação da maior influência da coordenação no processo de aprendizagem. Outro ponto dos experimentos seria a investigação do acompanhamento do professor no processo de busca e diálogo dos alunos. [Mayweg-Paus et al. 2020] destaca sobre a necessidade de maior investigação de quando ocorre o crescimento do conhecimento e de níveis cognitivos dos alunos durante a busca e a ontologia está preparada para mapear esses eventos. Como foi citado nos trabalhos relacionados, a área de PLN avança em compreender o que ocorre em diálogo dos alunos em fóruns e chats, podendo assim, ser combinado experimentos dessa área com uso da ontologia no contexto de CIS.

\section{Referências}

Alabri, A., Al-Khanjari, Z., Jamoussi, Y., and Kraiem, N. (2019). Mining the students' chat conversations in a personalized e-learning environment. International Journal of Emerging Technologies in Learning (iJET), 14(23):98-124.

Avià, L. (2019). Learn to work together: Trends in social and collaborative information seeking. Information and technology transforming lives: connection, interaction, innovation.

Basu, A. (2019). Semantic web, ontology, and linked data. In Web services: concepts, methodologies, tools, and applications, pages 127-148. IGI Global. 
Chi, Y., Han, S., He, D., and Meng, R. (2016). Exploring knowledge learning in collaborative information seeking process. In CEUR Workshop Proceedings.

Dionísio, M., Ferreira, R., Cavalcanti, A., Carvalho, R., and Neto, S. (2017). Mineração de texto aplicada à identificação de colaboração em fóruns educacionais. In Brazilian Symposium on Computers in Education (Simpósio Brasileiro de Informática na Educação-SBIE), volume 28, page 1437.

Fulantelli, G., Marenzi, I., Ahmad, Q. A. I., and Taibi, D. (2016). Sar-web-a tool to support search as learning processes. In SAL@SIGIR.

Haythornthwaite, C. (2019). Learning, connectivity and networks. Information and Learning Sciences.

Hertzum, M. and Hansen, P. (2019). Empirical studies of collaborative information seeking: a review of methodological issues. Journal of Documentation.

Hiltz, S. R. and Turoff, M. (2002). What makes learning networks effective? Communications of the ACM, 45(4):56-59.

Hong, S., Suh, M., Kim, T. S., Smoke, I., Sien, S., Ng, J., Zachry, M., and Kim, J. (2019). Design for collaborative information-seeking: Understanding user challenges and deploying collaborative dynamic queries. Proceedings of the ACM on HumanComputer Interaction, 3(CSCW):1-24.

Hoppe, A., Holtz, P., Kammerer, Y., Yu, R., Dietze, S., and Ewerth, R. (2018). Current challenges for studying search as learning processes. Proceedings of Learning and Education with Web Data, Amsterdam, Netherlands.

Hu, Z., Liskin, V., Syrota, S., Cholyshkina, O., and Seilova, N. (2019). Ontology-based model of information technology for e-learning systems. In COAPSN, pages 230-241.

Karanam, S. and van Oostendorp, H. (2016). Integrating domain knowledge differences into modeling user clicks on search result pages. In SAL@SIGIR.

Knight, S., Rienties, B., Littleton, K., Tempelaar, D., Mitsui, M., and Shah, C. (2017). The orchestration of a collaborative information seeking learning task. Information Retrieval Journal.

Konys, A. (2018). Knowledge systematization for ontology learning methods. Procedia computer science, 126:2194-2207.

Lebo, T., Sahoo, S., McGuinness, D., Belhajjame, K., Cheney, J., Corsar, D., Garijo, D., Soiland-Reyes, S., Zednik, S., and Zhao, J. (2013). Prov-o: The prov ontology. W3C recommendation, 30 .

Li, S., Abel, M.-H., and Negre, E. (2019). Towards a collaboration context ontology. In 2019 IEEE 23rd International Conference on Computer Supported Cooperative Work in Design (CSCWD), pages 93-98. IEEE.

Machado, M., Gimenez, P., and Siqueira, S. (2020). Raising the dimensions and variables for searching as a learning process: A systematic mapping of the literature. In Anais do XXXI Simpósio Brasileiro de Informática na Educação, pages 1393-1402, Porto Alegre, RS, Brasil. SBC. 
Machado, M., Pinelli, C., and Siqueira, S. (2019). A evolução da área de busca como um processo de aprendizagem com base em um mapeamento sistemático. In Anais dos Workshops do Congresso Brasileiro de Informática na Educação.

Mao, J., Liu, Y., Zhang, M., and Ma, S. (2016). How does domain expertise affect users' search processes in exploratory searches? In SAL@ SIGIR.

Mayweg-Paus, E., Zimmermann, M., Le, N.-T., and Pinkwart, N. (2020). A review of technologies for collaborative online information seeking: On the contribution of collaborative argumentation. Education and Information Technologies, pages 1-37.

Morris, M. R. and Teevan, J. (2009). Collaborative web search: Who, what, where, when, and why. Synthesis Lectures on Information Concepts, Retrieval, and Services.

Oktavia, T., Prabowo, H., Kosala, R., Supangkat, S. H., et al. (2016). A conceptual social learning ontology for higher education in e-learning 2.0. In 2016 International Conference on Information Management and Technology (ICIMTech), pages 165-170. IEEE.

Reynolds, R. B. (2016). Relationships among tasks, collaborative inquiry processes, inquiry resolutions, and knowledge outcomes in adolescents during guided discoverybased game design in school. Journal of Information Science.

Rieh, S. Y., Collins-Thompson, K., Hansen, P., and Lee, H.-J. (2016). Towards searching as a learning process: A review of current perspectives and future directions. Journal of Information Science.

Rikarno Putra, S. (2018). Evaluating collaborative search for a learning-oriented search task. Master's thesis, Delft University of Technology.

Souza, A. K., Guizzardi, R. S., Campos, M. L. M., and Guizzardi, G. (2016). Extending an ontology editor for domain-related ontology patterns reuse: An application in the collaboration domain. In ONTOBRAS, pages 47-58.

Stahl, G., Koschmann, T. D., and Suthers, D. D. (2006). Computer-supported collaborative learning. Cambridge handbook of the learning sciences.

Tibau, M., Siqueira, S., and Nunes, B. P. (2019). A comparison between entity-centric knowledge base and knowledge graph to represent semantic relationships for searching as learning situations. In Anais dos Workshops do Congresso Brasileiro de Informática na Educação, volume 8, page 823.

Tibau, M., Siqueira, S. W., Nunes, B. P., Bortoluzzi, M., and Marenzi, I. (2018). Modeling exploratory search as a knowledge-intensive process. In 2018 IEEE 18th International Conference on Advanced Learning Technologies (ICALT), pages 34-38. IEEE.

Vakkari, P. (2016). Searching as learning: A systematization based on literature. Journal of Information Science.

van Leeuwen, A. and Janssen, J. (2019). A systematic review of teacher guidance during collaborative learning in primary and secondary education. Educational Research Review, 27:71-89.

White, R. W. and Roth, R. A. (2009). Exploratory search: Beyond the query-response paradigm. Synthesis lectures on information concepts, retrieval, and services. 
Wu, D., Liang, S., and Yu, W. (2018). Collaborative information searching as learning in academic group work. Aslib Journal of Information Management.

Zachman, J. A. (1987). A framework for information systems architecture. IBM systems journal, 26(3):276-292. 